\title{
ON REAL CHAINS OF EVOLUTION ALGEBRAS
}

\author{
B.A. OMIROV, U.A. ROZIKOV, K. M. TULENBAYEV
}

\begin{abstract}
In this paper we define a chain of $n$-dimensional evolution algebras corresponding to a permutation of $n$ numbers. We show that a chain of evolution algebras (CEA) corresponding to a permutation is trivial (consisting only algebras with zeromultiplication) iff the permutation has not a fixed point. We show that a CEA is a chain of nilpotent algebras (independently on time) iff it is trivial. We construct a wide class of chains of 3 -dimensional EAs and a class of symmetric $n$-dimensional CEAs. A construction of arbitrary dimensional CEAs is given. Moreover, for a chain of 3-dimensional EAs we study the behavior of the baric property, the behavior of the set of absolute nilpotent elements and dynamics of the set of idempotent elements depending on the time.
\end{abstract}

AMS classifications (2010): 17D92; 17D99; 60J27

Keywords: Evolution algebra; time; Chapman-Kolmogorov equation; nilpotentcy.

\section{INTRODUCTION}

Evolutionary theory is important to a proper understanding of living populations at all levels. The paper [8] gives a comprehensive survey of problems in evolutionary theory studied by mathematical methods, both with regard to past developments, the present state of the subject and probable future trends. Many of the models considered are actually within the scope of mathematical population genetics, although the general ideas discussed have implications for a much wider area.

The formal language of abstract algebra to study of genetics was introduced in papers [4-6]. In recent years many authors have tried to investigate the difficult problem of classification of these algebras. The most comprehensive references for the mathematical research done in this area are $[2,9,10,13,14$.

In [9] an evolution algebra $\mathcal{A}$ associated with free population is introduced and using this non-associative algebra many results are obtained in explicit form, e.g. the explicit description of stationary quadratic operators, and the explicit solutions of a nonlinear evolutionary equation in the absence of selection, as well as general theorems on convergence to equilibrium in the presence of selection.

In [13] a new type of evolution algebra is introduced. This evolution algebra is defined as follows. Let $(E, \cdot)$ be an algebra over a field $K$. If it admits a basis $\left\{e_{1}, e_{2}, \ldots\right\}$ such that $e_{i} \cdot e_{j}=0$, if $i \neq j$ and $e_{i} \cdot e_{i}=\sum_{k} a_{i k} e_{k}$ for any $i$, then this algebra is called an evolution algebra. 
In this paper by the term evolution algebra we will understand a finite dimensional evolution algebra $E$ (as mentioned above) over the field $\mathbb{R}$.

In the book [13], the foundation of evolution algebra theory and applications in nonMendelian genetics and Markov chains are developed, with pointers to some further research topics.

In [11] the algebraic structures of function spaces defined by graphs and state spaces equipped with Gibbs measures by associating evolution algebras are studied. Results of [1] also allow a natural introduction of thermodynamics in studying of several systems of biology, physics and mathematics by theory of evolution algebras.x

Recently in 1 a notion of a chain of evolution algebras (CEA) is introduced. This chain is a dynamical system the state of which at each given time is an evolution algebra. The sequence of matrices of the structural constants for this chain of evolution algebras satisfies the Chapman-Kolmogorov equation. It is known that if a matrix satisfying the Chapman-Kolmogorov equation is stochastic, then it generates a Markov process. In [7] the authors investigated the time evolution of stochastic non-Markov processes as they occur in the coarse-grained description of open and closed systems. Some aspects of the theory are illustrated for the two-state process and the Gauss process. The CEA also can be motivated in a such way.

Following [1] we recall the definitions. Consider a family $\left\{E^{[s, t]}: s, t \in \mathbb{R}, 0 \leq s \leq t\right\}$ of $n$-dimensional evolution algebras over the field $\mathbb{R}$ with basis $\left\{e_{1}, \ldots, e_{n}\right\}$ and multiplication table

$$
e_{i} e_{i}=\sum_{j=1}^{n} a_{i j}^{[s, t]} e_{j}, \quad i=1, \ldots, n ; \quad e_{i} e_{j}=0, \quad i \neq j,
$$

where parameters $s, t$ are considered as time.

Denote by $\mathcal{M}^{[s, t]}=\left(a_{i j}^{[s, t]}\right)_{i, j=1, \ldots, n}$-the matrix of structural constants of $E^{[s, t]}$.

Definition 1. A family $\left\{E^{[s, t]}: s, t \in \mathbb{R}, 0 \leq s \leq t\right\}$ of $n$-dimensional evolution algebras over the field $\mathbb{R}$ is called a chain of evolution algebras if the matrix $\mathcal{M}^{[s, t]}$ of its structural constants satisfies the Chapman-Kolmogorov equation:

$$
\mathcal{M}^{[s, t]}=\mathcal{M}^{[s, \tau]} \mathcal{M}^{[\tau, t]}, \text { for any } s<\tau<t .
$$

Definition 2. A CEA is called a time-homogenous $C E A$ if the matrix $\mathcal{M}^{[s, t]}$ depends only on $t-s$. In this case we write $\mathcal{M}^{[t-s]}$.

In 12 25 distinct examples of chains of two-dimensional evolution algebras are constructed. For all of chains constructed in [1] and 12 the behavior of the baric property, the behavior of the set of absolute nilpotent elements and dynamics of the set of idempotent elements depending on the time are studied.

The paper is organized as follows. In Section 2 we define a chain of $n$-dimensional evolution algebras corresponding to a permutation of $n$ numbers. We show that a CEA corresponding to a permutation is trivial (consisting only algebras with zero-products) iff the permutation has not a fixed point. In Section 3 we show that a CEA is a chain of nilpotent algebras (independently on time) iff it is trivial. Section 4 is devoted to a 
construction of chains of 3-dimensional EAs. In Section 5 for a chain of 3-dimensional EAs we study the behavior of the baric property, the behavior of the set of absolute nilpotent elements and dynamics of the set of idempotent elements depending on the time. Section 6 is devoted to construction of an arbitrary dimensional symmetric CEAs. In the last section we give an argument to construct a wide class of chains of arbitrary finite dimensional EAs.

\section{The CEA CORRESPonding to A PeRmutation}

Let $S_{n}$ be the group of permutations of the set $\{1,2, \ldots, n\}$. For a quadratic matrix $A=\left\{a_{i j}\right\}_{i, j=1}^{n}$ we say that it corresponds to a permutation $\pi$ if

$$
a_{i j}=\left\{\begin{array}{l}
0, \text { if } j \neq \pi(i) \\
\neq 0, \text { if } j=\pi(i) .
\end{array}\right.
$$

Let $A_{\pi}, B_{\pi}$ be two matrices corresponding to a permutation $\pi$. It is easy to see that

$$
A_{\pi} B_{\pi}=C_{\pi^{2}} .
$$

For a fixed $\pi \in S_{n}$ we shall construct CEAs corresponding to $\mathcal{M}_{\pi}^{[s, t]}$.

Theorem 1. Let $\pi \in S_{n}$ and $\left\{\mathcal{M}_{\pi}^{[s, t]}, \quad 0 \leq s \leq t\right\}$ be a family of matrices which satisfies the equation (1.2). Then

(i) $\mathcal{M}_{\pi}^{[s, t]} \equiv 0$ if $\{i: \pi(i)=i\}=\emptyset$.

(ii) $\mathcal{M}_{\pi}^{[s, t]} \neq 0$ if $\{i: \pi(i)=i\} \neq \emptyset$.

Proof. (i) Let $\mathcal{M}_{\pi}^{[s, \tau]} \mathcal{M}_{\pi}^{[\tau, t]}=\left(c_{i j}\right)_{i, j=1}^{n}$. We have by (2.2) that

$$
c_{i j}=\left\{\begin{array}{l}
0, \text { if } j \neq \pi^{2}(i) \\
\neq 0, \text { if } j=\pi^{2}(i) .
\end{array}\right.
$$

From (1.2) we get

$$
\left\{\begin{array}{l}
0 \neq c_{i \pi^{2}(i)}=a_{i \pi(i)}^{[s, \tau]} a_{\pi(i) \pi(\pi(i))}^{[\tau, t]}=a_{i \pi(i)}^{[s, t]}, \\
a_{i j}^{[s, t]}=0, \quad \text { if } j \neq \pi(i), \quad \forall s, t .
\end{array}\right.
$$

Hence $a_{i \pi(i)}^{[s, t]}=0$ iff $\pi(i) \neq \pi^{2}(i)$, i.e., $i \neq \pi(i)$.

(ii) Now assume $\{i: \pi(i)=i\} \neq \emptyset$. Without loss of generality we can take $\{i: \pi(i)=$ $i\}=\{1,2, \ldots, k\}$. Then from (2.4) we obtain

$$
a_{j j}^{[s, \tau]} a_{j j}^{[\tau, t]}=a_{j j}^{[s, t]}, \quad j=1, \ldots, k .
$$

This equation is known as Cantor's second equation which has very rich family of solutions:

\footnotetext{
a) $a_{j j}^{[s, t]}=\frac{\Phi_{j}(t)}{\Phi_{j}(s)}$, where $\Phi_{j}$ is an arbitrary function with $\Phi_{j}(s) \neq 0$;
} 
b)

$$
a_{j j}^{[s, t]}=\left\{\begin{array}{ll}
1, & \text { if } s \leq t<a_{j}, \\
0, & \text { if } t \geq a_{j},
\end{array} \quad \text { where } a_{j}>0 .\right.
$$

The CEAs $E_{\pi}^{[s, t]}$ corresponding to solutions a)-b) have matrix of structural constants in the following form:

$$
\mathcal{M}_{\pi}^{[s, t]}=\left(\begin{array}{cccccc}
a_{11}^{[s, t]} & 0 & 0 & \ldots & \ldots & 0 \\
0 & a_{22}^{[s, t]} & 0 & \ldots & \ldots & 0 \\
\vdots & \ldots & \ldots & \ldots & & \vdots \\
0 & 0 & \ldots & a_{k k}^{[s, t]} & \ldots & 0 \\
0 & 0 & \ldots & 0 & \ldots & 0 \\
\vdots & \ldots & \ldots & \ldots & & \vdots \\
0 & 0 & \ldots & 0 & \ldots & 0
\end{array}\right)
$$

This completes the proof.

\section{Nilpotent CEAS}

Definition 3. An element $x$ of an algebra $\mathcal{A}$ is called nil if there exists $n(a) \in \mathbb{N}$ such that $(\cdots \underbrace{((x \cdot x) \cdot x) \cdots x}_{n(a)})=0$. Algebra $\mathcal{A}$ is called nil if every element of the algebra is nil.

For $k \geq 1$ we introduce the following sequences:

$$
\begin{gathered}
\mathcal{A}^{(1)}=\mathcal{A}, \quad \mathcal{A}^{(k+1)}=\mathcal{A}^{(k)} \mathcal{A}^{(k)} . \\
\mathcal{A}^{<1>}=\mathcal{A}, \quad \mathcal{A}^{<k+1>}=\mathcal{A}^{<k>} \mathcal{A} . \\
\mathcal{A}^{1}=\mathcal{A}, \quad \mathcal{A}^{k}=\sum_{i=1}^{k-1} \mathcal{A}^{i} \mathcal{A}^{k-i} .
\end{gathered}
$$

Definition 4. An algebra $\mathcal{A}$ is called

(i) solvable if there exists $n \in \mathbb{N}$ such that $\mathcal{A}^{(n)}=0$ and the minimal such number is called index of solvability;

(ii) right nilpotent if there exists $n \in \mathbb{N}$ such that $\mathcal{A}^{<n>}=0$ and the minimal such number is called index of right nilpotency;

(iii) nilpotent if there exists $n \in \mathbb{N}$ such that $\mathcal{A}^{n}=0$ and the minimal such number is called index of nilpotency.

The following theorem is known 
Theorem 2. [2], [3] The following statements are equivalent for an n-dimensional evolution algebra $E$ :

a) The matrix corresponding to $E$ can be written as

$$
\widehat{A}=\left(\begin{array}{ccccc}
0 & a_{12} & a_{13} & \ldots & a_{1 n} \\
0 & 0 & a_{23} & \ldots & a_{2 n} \\
0 & 0 & 0 & \ldots & a_{3 n} \\
\vdots & \vdots & \vdots & \ldots & \vdots \\
0 & 0 & 0 & \ldots & 0
\end{array}\right)
$$

b) $E$ is a right nilpotent algebra;

c) $E$ is a nil algebra.

d) $E$ is a nilpotent algebra.

This section is devoted to the following

Theorem 3. A chain of evolution algebras $E^{[s, t]}$ is nilpotent for any $(s, t)$ if and only if $\mathcal{M}^{[s, t]}=0$ for any $(s, t)$.

Proof. Necessity. Let

$$
\mathcal{M}^{[s, t]}=\left(\begin{array}{ccccc}
0 & a_{12}^{[s, t]} & a_{13}^{[s, t]} & \ldots & a_{1 n}^{[s, t]} \\
0 & 0 & a_{23}^{[s, t]} & \ldots & a_{2 n}^{[s, t]} \\
0 & 0 & 0 & \ldots & a_{3 n}^{[s, t]} \\
\vdots & \vdots & \vdots & \ldots & \vdots \\
0 & 0 & 0 & \ldots & 0
\end{array}\right)
$$

From equation (1.2) we get

$$
\mathcal{M}^{[s, t]}=\left(\begin{array}{cccccc}
0 & 0 & a_{13}^{[s, t]} & a_{14}^{[s, t]} & \ldots & a_{1 n}^{[s, t]} \\
0 & 0 & 0 & a_{24}^{[s, t]} & \ldots & a_{2 n}^{[s, t]} \\
0 & 0 & 0 & 0 & \ldots & a_{3 n}^{[s, t]} \\
\vdots & \vdots & \vdots & \ldots & \vdots & \\
0 & 0 & 0 & 0 & \ldots & 0
\end{array}\right) .
$$

Iterating we get $\mathcal{M}^{[s, t]}=0$.

Sufficiency. Straightforward. 


\section{A construction of Chains of three-Dimensional EAs}

In this section we shall construct several new chains of three-dimensional evolution algebras. Consider a matrix of structural constants having the form

$$
\mathcal{M}^{[s, t]}=\left(\begin{array}{ccc}
a_{1}^{[s, t]} & a_{2}^{[s, t]} & a_{3}^{[s, t]} \\
0 & a_{4}^{[s, t]} & a_{5}^{[s, t]} \\
0 & 0 & a_{6}^{[s, t]}
\end{array}\right) .
$$

Then the equation (1.2) becomes:

$$
\begin{aligned}
& a_{1}^{[s, \tau]} a_{1}^{[\tau, t]}=a_{1}^{[s, t]} \\
& a_{1}^{[s, \tau]} a_{2}^{[\tau, t]}+a_{2}^{[s, \tau]} a_{4}^{[\tau, t]}=a_{2}^{[s, t]} \\
& a_{1}^{[s, \tau]} a_{3}^{[\tau, t]}+a_{2}^{[s, \tau]} a_{5}^{[\tau, t]}+a_{3}^{[s, \tau]} a_{6}^{[\tau, t]}=a_{3}^{[s, t]} \\
& a_{4}^{[s, \tau]} a_{4}^{[\tau, t]}=a_{4}^{[s, t]} \\
& a_{4}^{[s, \tau]} a_{5}^{[\tau, t]}+a_{5}^{[s, \tau]} a_{6}^{[\tau, t]}=a_{5}^{[s, t]} \\
& a_{6}^{[s, \tau]} a_{6}^{[\tau, t]}=a_{6}^{[s, t]} .
\end{aligned}
$$

We note that the first, the fourth and the last equations of the system (4.2) are Cantor's second equations and they have the following solutions $(i=1,4,6)$ :

$\left(1_{i}\right)$

$$
a_{i}^{[s, t]}=\frac{\Phi_{i}(t)}{\Phi_{i}(s)}
$$

where $\Phi_{i}$ is an arbitrary function with $\Phi_{i}(s) \neq 0, i=1,4,6$;

$\left(2_{i}\right)$

$$
a_{i}^{[s, t]}=\left\{\begin{array}{ll}
1, & \text { if } s \leq t<\alpha_{i}, \\
0, & \text { if } t \geq \alpha_{i},
\end{array} \quad \text { where } \alpha_{i}>0, i=1,4,6 .\right.
$$

Thus we have eight possibilities for $\left(a_{1}^{[s, t]}, a_{4}^{[s, t]}, a_{6}^{[s, t]}\right)$. Consider these possibilities:

Case $\left(1_{1}\right),\left(1_{4}\right),\left(1_{6}\right)$ : The second equation of (4.2) becomes

$$
\frac{\Phi_{1}(\tau)}{\Phi_{1}(s)} a_{2}^{[\tau, t]}+\frac{\Phi_{4}(t)}{\Phi_{4}(\tau)} a_{2}^{[s, \tau]}=a_{2}^{[s, t]} .
$$

From this equation denoting $\gamma(s, t)=\frac{\Phi_{1}(s)}{\Phi_{4}(t)} a_{2}^{[s, t]}$ we get

$$
\gamma(s, t)=\gamma(s, \tau)+\gamma(\tau, t) .
$$

This equation is known as Cantor's first equation which has solutions

$$
\gamma(s, t)=\xi(t)-\xi(s) \text {, where } \xi \text { is an arbitrary function. }
$$

Hence

$$
a_{2}^{[s, t]}=\frac{\Phi_{4}(t)}{\Phi_{1}(s)}(\xi(t)-\xi(s))
$$


Similarly, one gets that

$$
a_{5}^{[s, t]}=\frac{\Phi_{6}(t)}{\Phi_{4}(s)}(f(t)-f(s)),
$$

where $f$ is an arbitrary function. Using these functions from the third equation of (4.2) we obtain

$$
\varphi^{[s, \tau]}+\varphi^{[\tau, t]}=\varphi^{[s, t]}+(f(\tau)-f(t))(\xi(\tau)-\xi(s)),
$$

where $\varphi^{[s, t]}=\frac{\Phi_{1}(s)}{\Phi_{6}(t)} a_{3}^{[s, t]}$. We shall find solutions to (4.7) which have the form

$$
\varphi^{[s, t]}=a f(s) \xi(s)+b f(s) \xi(t)+c f(t) \xi(s)+d f(t) \xi(t) .
$$

Substituting this function in (4.7) we obtain $b=0, c=-1$ and $a+d=1$. Hence the following function is a solution to (4.7):

$$
\varphi_{0}^{[s, t]}=a f(s) \xi(s)-f(t) \xi(s)+(1-a) f(t) \xi(t),
$$

where $a \in \mathbb{R}$. Moreover, it is easy to see that $\varphi^{[s, t]}=\varphi_{0}^{[s, t]}+\gamma(t)-\gamma(s)$ is also solution to (4.7), where $\gamma$ is an arbitrary function.

Consequently,

$$
a_{3}^{[s, t]}=\frac{\Phi_{6}(t)}{\Phi_{1}(s)}[a f(s) \xi(s)-f(t) \xi(s)+(1-a) f(t) \xi(t)+\gamma(t)-\gamma(s)] .
$$

Thus we have proved the following

Proposition 1. The matrix (4.1) with elements given by (4.3), 4.5), (4.6) and (4.8) generates a CEA.

Case $\left(1_{1}\right),\left(1_{4}\right),\left(2_{6}\right)$ : In this case the functions $a_{i}^{[s, t]}, i=1,2,4$ coincide with functions given in the previous case. The function $a_{6}^{[s, t]}$ is given by (4.4). Here we have to find $a_{3}^{[s, t]}$ and $a_{5}^{[s, t]}$. From (4.2) we have

$$
a_{5}^{[s, t]}=\left\{\begin{array}{l}
a_{5}^{[s, \tau]}+\frac{\Phi_{4}(\tau)}{\Phi_{4}(s)} a_{5}^{[\tau, t]}, \quad \text { if } s \leq t<\alpha_{6} \\
\frac{\Phi_{4}(\tau)}{\Phi_{4}(s)} a_{5}^{[\tau, t]}, \quad \text { if } t \geq \alpha_{6} .
\end{array}\right.
$$

Denoting $\psi^{[s, t]}=\Phi_{4}(s) a_{5}^{[s, t]}$ from (4.9) we get

$$
\psi^{[s, t]}=\left\{\begin{array}{l}
\psi^{[s, \tau]}+\psi^{[\tau, t]}, \quad \text { if } s \leq t<\alpha_{6} \\
\psi^{[\tau, t]}, \quad \text { if } t \geq \alpha_{6} .
\end{array}\right.
$$

This equation has the following solution

$$
\psi^{[s, t]}=\left\{\begin{array}{l}
\beta(t)-\beta(s), \quad \text { if } s \leq t<\alpha_{6} \\
\gamma(t), \quad \text { if } t \geq \alpha_{6},
\end{array}\right.
$$


where $\beta$ and $\gamma$ are arbitrary functions. Therefore, we have

$$
a_{5}^{[s, t]}=\frac{1}{\Phi_{4}(s)}\left\{\begin{array}{l}
\beta(t)-\beta(s), \quad \text { if } s \leq t<\alpha_{6} \\
\gamma(t), \quad \text { if } t \geq \alpha_{6}
\end{array}\right.
$$

Now for $a_{3}^{[s, t]}$ we have

$$
a_{3}^{[s, t]}=\frac{1}{\Phi_{1}(s)}\left\{\begin{array}{l}
\Phi_{1}(s) a_{3}^{[s, \tau]}+\Phi_{1}(\tau) a_{3}^{[\tau, t]}+(\xi(\tau)-\xi(s))(\beta(t)-\beta(\tau)), \quad \text { if } s \leq t<\alpha_{6} \\
\Phi_{1}(\tau) a_{3}^{[\tau, t]}+(\xi(\tau)-\xi(s)) \gamma(t), \quad \text { if } t \geq \alpha_{6} .
\end{array}\right.
$$

Denoting $\eta(s, t)=\Phi_{1}(s) a_{3}^{[s, t]}$ and using arguments of the previous case we get

$$
\eta(s, t)=\left\{\begin{array}{l}
a \beta(s) \xi(s)-\beta(t) \xi(s)+(1-a) \beta(t) \xi(t)+\theta(t)-\theta(s), \quad \text { if } s \leq t<\alpha_{6} \\
\gamma(t)(v(t)+b \xi(t)-\xi(s)), \quad \text { if } t \geq \alpha_{6},
\end{array}\right.
$$

where $b \in \mathbb{R}$ and $\theta, v$ are arbitrary functions. Consequently,

$$
a_{3}^{[s, t]}=\frac{1}{\Phi_{1}(s)}\left\{\begin{array}{l}
a \beta(s) \xi(s)-\beta(t) \xi(s)+(1-a) \beta(t) \xi(t)+\theta(t)-\theta(s), \quad \text { if } \quad s \leq t<\alpha_{6} \\
\gamma(t)(v(t)+b \xi(t)-\xi(s)), \quad \text { if } t \geq \alpha_{6},
\end{array}\right.
$$

Thus we have proved the following

Proposition 2. The matrix (4.1) with elements given by (4.3) for $i=1,4$; (4.4) for $i=6$; 4.5), 4.10) and (4.12) generates a CEA.

Case $\left(1_{1}\right),\left(2_{4}\right),\left(2_{6}\right)$ : From the second equation of (4.2) we get

$$
a_{2}^{[s, t]}=\frac{1}{\Phi_{1}(s)}\left\{\begin{array}{l}
\omega(t)-\omega(s), \quad \text { if } s \leq t<\alpha_{4} \\
\delta(t), \quad \text { if } t \geq \alpha_{4}
\end{array}\right.
$$

where $\omega$ and $\delta$ are arbitrary functions.

Now we shall find $a_{5}^{[s, t]}$. Without loss of generality we assume $\alpha_{4}<\alpha_{6}$. Then we obtain the following equation

$$
a_{5}^{[s, t]}=\left\{\begin{array}{l}
a_{5}^{[s, \tau]}+a_{5}^{[\tau, t]}, \quad \text { if } s \leq \tau<\min \left\{\alpha_{4}, t\right\} \\
a_{5}^{[s, \tau]}, \quad \text { if } \alpha_{4} \leq \tau<t<\alpha_{6} \\
0, \quad \text { if } \quad \alpha_{4} \leq \tau, \quad \alpha_{6} \leq t .
\end{array}\right.
$$

This equation has the following solutions

$$
a_{5}^{[s, t]}=\left\{\begin{array}{l}
\zeta(t)-\zeta(s), \text { if } s \leq \tau<\min \left\{\alpha_{4}, t\right\} \\
p(s), \quad \text { if } \alpha_{4} \leq \tau<t<\alpha_{6} \\
0, \quad \text { if } \alpha_{4} \leq \tau, \quad \alpha_{6} \leq t
\end{array}\right.
$$


where $\zeta$ and $p$ are arbitrary functions.

In this case $a_{3}^{[s, t]}$ satisfies the following equation

$$
a_{3}^{[s, t]}=\left\{\begin{array}{l}
a_{3}^{[s, \tau]}+\frac{\Phi_{1}(\tau)}{\Phi_{1}(s)} a_{3}^{[\tau, t]}+\frac{1}{\Phi_{1}(s)}(\omega(\tau)-\omega(s))(\zeta(t)-\zeta(\tau)), \quad \text { if } s \leq \tau<\min \left\{\alpha_{4}, t\right\} \\
a_{3}^{[s, \tau]}+\frac{\Phi_{1}(\tau)}{\Phi_{1}(s)} a_{3}^{[\tau, t]}+\frac{1}{\Phi_{1}(s)} \delta(\tau) p(\tau), \quad \text { if } \quad \alpha_{4} \leq \tau<t<\alpha_{6} \\
\frac{\Phi_{1}(\tau)}{\Phi_{1}(s)} a_{3}^{[\tau, t]}, \quad \text { if } \quad \alpha_{4} \leq \tau, \quad \alpha_{6} \leq t .
\end{array}\right.
$$

Similarly as above one can see that this equation has the following solution

$$
a_{3}^{[s, t]}=\frac{1}{\Phi_{1}(s)}\left\{\begin{array}{l}
a \zeta(s) \omega(s)-\zeta(t) \omega(s)+(1-a) \zeta(t) \omega(t)+d(t)-d(s), \quad \text { if } \quad s \leq \tau<\min \left\{\alpha_{4}, t\right\} \\
a \delta(s) p(s)-(1+a) \delta(t) p(t)+e(t)-e(s), \quad \text { if } \quad \alpha_{4} \leq \tau<t<\alpha_{6}, \\
m(t), \quad \text { if } \alpha_{4} \leq \tau, \quad \alpha_{6} \leq t,
\end{array}\right.
$$

where $d, e$ and $m$ are arbitrary functions, $a \in \mathbb{R}$.

Thus we have proved the following

Proposition 3. The matrix (4.1) with elements given by 4.3) for $i=1$; 4.4) for $i=4,6$; 4.13), 4.14) and 4.15) generates a CEA.

Case $\left(2_{1}\right),\left(2_{4}\right),\left(2_{6}\right)$ : Assume $\alpha_{1}<\alpha_{4}<\alpha_{6}$. In this case for $a_{2}^{[s, t]}$ we have

$$
a_{2}^{[s, t]}=\left\{\begin{array}{l}
a_{2}^{[s, \tau]}+a_{2}^{[\tau, t]}, \quad \text { if } s \leq \tau<\min \left\{\alpha_{1}, t\right\} \\
a_{2}^{[s, \tau]}, \quad \text { if } \alpha_{1} \leq \tau<t<\alpha_{4} \\
0, \quad \text { if } \quad \alpha_{1} \leq \tau, \quad \alpha_{4} \leq t
\end{array}\right.
$$

This has the following solution

$$
a_{2}^{[s, t]}=\left\{\begin{array}{l}
l(t)-l(s), \quad \text { if } s \leq \tau<\min \left\{\alpha_{1}, t\right\} \\
w(s), \quad \text { if } \alpha_{1} \leq \tau<t<\alpha_{4} \\
0, \quad \text { if } \alpha_{1} \leq \tau, \quad \alpha_{4} \leq t
\end{array}\right.
$$

where $l$ and $w$ are arbitrary functions.

Similarly

$$
a_{5}^{[s, t]}=\left\{\begin{array}{l}
A(t)-A(s), \quad \text { if } s \leq \tau<\min \left\{\alpha_{4}, t\right\} \\
B(s), \quad \text { if } \alpha_{4} \leq \tau<t<\alpha_{6} \\
0, \quad \text { if } \alpha_{4} \leq \tau, \quad \alpha_{6} \leq t,
\end{array}\right.
$$


where $A$ and $B$ are arbitrary functions. For $a_{3}^{[s, t]}$ we have the following equation

$$
a_{3}^{[s, t]}=\left\{\begin{array}{l}
a_{3}^{[s, \tau]}+a_{3}^{[\tau, t]}+(l(\tau)-l(s))(A(t)-A(\tau)), \quad \text { if } s \leq \tau<\min \left\{\alpha_{1}, t\right\} \\
a_{3}^{[s, \tau]}+w(s)(A(t)-A(\tau)), \quad \text { if } \alpha_{1} \leq \tau<t<\alpha_{4} \\
a_{3}^{[\tau, t]}, \quad \text { if } \alpha_{4} \leq t<\alpha_{6} \\
0, \quad \text { if } t \geq \alpha_{6} .
\end{array}\right.
$$

This equation has the following solution

$$
a_{3}^{[s, t]}=\left\{\begin{array}{l}
a A(s) l(s)-A(t) l(s)+(1-a) A(t) l(t)+c(t)-c(s), \text { if } s \leq \tau<\min \left\{\alpha_{1}, t\right\} \\
(A(t)+b A(s)+g(s)) w(s), \text { if } \alpha_{1} \leq \tau<t<\alpha_{4}, \\
q(s), \text { if } \alpha_{4} \leq t<\alpha_{6} \\
0, \text { if } t \geq \alpha_{6},
\end{array}\right.
$$

where $c, g$ and $q$ are arbitrary functions, $a, b \in \mathbb{R}$.

Thus we have proved the following

Proposition 4. The matrix (4.1) with elements given by (4.4) for $i=1,4,6$; (4.16), 4.17) and 4.18) generates a CEA.

Remark 1. We note that the cases $\left\{\left(1_{1}\right),\left(2_{4}\right),\left(1_{6}\right)\right\},\left\{\left(2_{1}\right),\left(1_{4}\right),\left(1_{6}\right)\right\}$ are similar to the case $\left\{\left(1_{1}\right),\left(1_{4}\right),\left(2_{6}\right)\right\}$ and $\left\{\left(2_{1}\right),\left(2_{4}\right),\left(1_{6}\right)\right\},\left\{\left(2_{1}\right),\left(1_{4}\right),\left(2_{6}\right)\right\}$ are similar to the case $\left\{\left(1_{1}\right),\left(2_{4}\right),\left(2_{6}\right)\right\}$. Hence these cases do not give any new CEA.

\section{Dynamical properties of CEAs given by matrix (4.1)}

In this section we consider CEAs constructed by matrix (4.1). To avoid many special cases we assume

$$
\operatorname{det}\left(\mathcal{M}^{[s, t]}\right)=a_{1}^{[s, t]} a_{4}^{[s, t]} a_{6}^{[s, t]} \neq 0 \text { for all }(s, t) .
$$

We note that the CEAs given in Proposition 1 satisfy the condition (5.1).

In [1] a notion of property transition for CEAs is defined. We recall the definitions:

Definition 5. Assume a $C E A, E^{[s, t]}$, has a property, say $P$, at pair of times $\left(s_{0}, t_{0}\right)$; one says that the CEA has $P$ property transition if there is a pair $(s, t) \neq\left(s_{0}, t_{0}\right)$ at which the CEA has no the property $P$.

Denote

$$
\begin{gathered}
\mathcal{T}=\{(s, t): 0 \leq s \leq t\} \\
\mathcal{T}_{P}=\left\{(s, t) \in \mathcal{T}: E^{[s, t]} \text { has property } P\right\} \\
\mathcal{T}_{P}^{0}=\mathcal{T} \backslash \mathcal{T}_{P}=\left\{(s, t) \in \mathcal{T}: E^{[s, t]} \text { has no property } P\right\}
\end{gathered}
$$

The sets have the following meaning

$\mathcal{T}_{P}$-the duration of the property $P$; 
$\mathcal{T}_{P}^{0}$-the lost duration of the property $P$;

A character for an algebra $A$ is a nonzero multiplicative linear form on $A$, that is, a nonzero algebra homomorphism from $A$ to $\mathbb{R}[9]$. Not every algebra admits a character. For example, an algebra with the zero multiplication has no character.

Definition 6. A pair $(A, \sigma)$ consisting of an algebra $A$ and a character $\sigma$ on $A$ is called a baric algebra. The homomorphism $\sigma$ is called the weight (or baric) function of $A$ and $\sigma(x)$ the weight (baric value) of $x$.

Recall that the element $x=\sum_{i=1}^{n} x_{i} e_{i}$ of an algebra $A$ is called an absolute nilpotent if $x^{2}=0$. For $n$-dimensional evolution algebra $x^{2}=0$ is given by the following system

$$
\sum_{i=1}^{n} a_{i j} x_{i}^{2}=0, \quad j=1, \ldots, n .
$$

An element $x$ of an algebra $\mathcal{A}$ is called idempotent if $x^{2}=x$. We denote by $\mathcal{I} d(\mathcal{A})$ the set of idempotent elements of an algebra $\mathcal{A}$. For an evolution algebra the equation $x^{2}=x$ can be written as

$$
x_{j}=\sum_{i=1}^{n} a_{i j} x_{i}^{2}, \quad j=1, \ldots, n .
$$

Now we are ready to formulate the following

Theorem 4. If a CEA, $E^{[s, t]}$ is defined by a matrix (4.1) satisfying (5.1) then

1. The algebra $E^{[s, t]}$ is baric for any $(s, t) \in \mathcal{T}$.

2. The algebra $E^{[s, t]}$ has a unique absolute nilpotent element $x=0$ for any $(s, t) \in \mathcal{T}$. 3 .

$$
\begin{gathered}
\mathcal{I} d\left(E^{[s, t]}\right)=\left\{\lambda_{1}, \lambda_{2}\right\} \bigcup\left\{\begin{array}{c}
\left\{\lambda_{3}\right\}, \quad \text { if } \quad(s, t) \in\left\{(s, t) \in \mathcal{T}: D_{1}(s, t)=0\right\} \\
\left\{\lambda_{4}, \lambda_{5}\right\}, \quad \text { if } \quad(s, t) \in\left\{(s, t) \in \mathcal{T}: D_{1}(s, t)>0\right\}
\end{array}\right. \\
\left\{\begin{array}{c}
\left\{\lambda_{6}\right\}, \quad \text { if } \quad(s, t) \in\left\{(s, t) \in \mathcal{T}: D_{2}(s, t)=D_{3}(s, t)=0\right\} \\
\left\{\lambda_{7}, \lambda_{8}\right\}, \quad \text { if } \quad(s, t) \in\left\{(s, t) \in \mathcal{T}: D_{2}(s, t)=0, D_{3}(s, t)>0\right\} \\
\left\{\lambda_{9}\right\}, \quad \text { if } \quad(s, t) \in\left\{(s, t) \in \mathcal{T}: D_{2}(s, t)>0, D_{4}(s, t)=0\right\} \\
\left\{\lambda_{10}, \lambda_{11}\right\}, \quad \text { if } \quad(s, t) \in\left\{(s, t) \in \mathcal{T}: D_{2}(s, t)>0, D_{4}(s, t)>0\right\} \\
\left\{\lambda_{12}\right\}, \quad \text { if } \quad(s, t) \in\left\{(s, t) \in \mathcal{T}: D_{2}(s, t)>0, D_{5}(s, t)=0\right\} \\
\left\{\lambda_{13}, \lambda_{14}\right\}, \quad \text { if } \quad(s, t) \in\left\{(s, t) \in \mathcal{T}: D_{2}(s, t)>0, D_{5}(s, t)>0\right\},
\end{array}\right. \\
\text { where } \lambda_{1}=(0,0,0), \lambda_{2}=\left(0,0,1 / a_{6}^{[s, t]}\right), \lambda_{3}=\left(0,1 / a_{4}^{[s, t]}, 1 /\left(2 a_{6}^{[s, t]}\right)\right),
\end{gathered}
$$$$
D_{1}(s, t)=1-\frac{4 a_{5}^{[s, t]} a_{6}^{[s, t]}}{\left(a_{4}^{[s, t]}\right)^{2}}, \quad \lambda_{4,5}=\left(0, \frac{1}{a_{4}^{[s, t]}}, \frac{1 \pm \sqrt{D_{1}(s, t)}}{2 a_{6}^{[s, t]}}\right),
$$$$
D_{2}(s, t)=1-\frac{4 a_{2}^{[s, t]} a_{4}^{[s, t]}}{\left(a_{1}^{[s, t]}\right)^{2}}, \quad D_{3}(s, t)=1-4 a_{6}^{[s, t]}\left(\frac{a_{3}^{[s, t]}}{\left(a_{1}^{[s, t]}\right)^{2}}+\frac{a_{5}^{[s, t]}}{\left(2 a_{4}^{[s, t]}\right)^{2}}\right) \text {, }
$$ 


$$
\begin{gathered}
\lambda_{6}=\left(\frac{1}{a_{1}^{[s, t]}}, \frac{1}{2 a_{4}^{[s, t]}}, \frac{1}{2 a_{6}^{[s, t]}}\right), \quad \lambda_{7,8}=\left(\frac{1}{a_{1}^{[s, t]}}, \frac{1}{2 a_{4}^{[s, t]}}, \frac{1 \pm \sqrt{D_{3}(s, t)}}{2 a_{6}^{[s, t]}}\right), \\
D_{4,5}(s, t)=1-4 a_{6}^{[s, t]}\left(\frac{a_{3}^{[s, t]}}{\left(a_{1}^{[s, t]}\right)^{2}}+a_{5}^{[s, t]} y_{4,5}^{2}\right), \text { with } y_{4,5}=\frac{1 \pm \sqrt{D_{2}(s, t)}}{2 a_{4}^{[s, t]}}, \\
\lambda_{9}=\left(\frac{1}{a_{1}^{[s, t]}}, y_{4}, \frac{1}{2 a_{6}^{[s, t]}}\right), \quad \lambda_{10,11}=\left(\frac{1}{a_{1}^{[s, t]}}, y_{4}, \frac{1 \pm \sqrt{D_{4}(s, t)}}{2 a_{6}^{[s, t]}}\right) \\
\lambda_{12}=\left(\frac{1}{a_{1}^{[s, t]}}, y_{5}, \frac{1}{2 a_{6}^{[s, t]}}\right), \quad \lambda_{13,14}=\left(\frac{1}{a_{1}^{[s, t]}}, y_{5}, \frac{1 \pm \sqrt{D_{5}(s, t)}}{2 a_{6}^{[s, t]}}\right)
\end{gathered}
$$

Proof. 1. Under condition (5.1) this assertion follows from [1, Theorem 3.2].

2. From condition (5.1) and system (5.2) we conclude that $x=(0,0,0)$ is the unique solution.

3. For matrix (4.1) the system (5.3) can be written as

$$
\begin{aligned}
& x_{1}=a_{1}^{[s, t]} x_{1}^{2} \\
& x_{2}=a_{2}^{[s, t]} x_{1}^{2}+a_{4}^{[s, t]} x_{2}^{2} \\
& x_{3}=a_{3}^{[s, t]} x_{1}^{2}+a_{5}^{[s, t]} x_{2}^{2}+a_{6}^{[s, t]} x_{3}^{2} .
\end{aligned}
$$

Hence for $x_{1}$ there is two possibilities $x_{1}=0$ and $x_{1}=1 / a_{1}^{[s, t]}$. For each value of $x_{1}$ the $x_{2}$ also has up to two possible values. For fixed values of $x_{1}$ and $x_{2}$ we have two possible values for $x_{3}$. Carefully computing the values gives the result.

Corollary 1. The CEA considered in Theorem 4 has the following dynamics:

1. It has not "baric" property transition.

2. It has not "uniqueness absolute nilpotent element" property transition.

3. It has "a fixed number of idempotent elements" property transition. The transition critical sets are given by $\left\{(s, t): D_{i}(s, t)=0\right\}, i=1,2,3,4,5$, i.e. the number of idempotent elements changes depending on time, when the time point crosses these sets.

\section{CEAs CORRESPONDING TO SYMMETRIC MATRICES}

In this section we consider $\mathcal{M}^{[s, t]}=\left(a_{i j}^{[s, t]}\right)_{i, j=1, \ldots, n}$ with $a_{i j}^{[s, t]}=a_{j i}^{[s, t]}$ and solve the equation (1.2) for such matrices. The equation (1.2) has the following form

$$
\sum_{k=1}^{n} a_{i k}^{[s, \tau]} a_{k j}^{[\tau, t]}=a_{i j}^{[s, t]}, \quad i, j=1, \ldots, n .
$$

We introduce the following functions

$$
f_{i}(s, t)=\sum_{j=1}^{n} a_{i j}^{[s, t]}, \quad i=1, \ldots, n .
$$




$$
g_{i k}(s, t)=\sum_{j=1}^{n} a_{i j}^{[s, t]}-a_{i k}^{[s, t]}, \quad i, k=1, \ldots, n .
$$

Using (6.1) from (6.2) we get

$$
f_{i}(s, t)=\sum_{j=1}^{n} \sum_{k=1}^{n} a_{i k}^{[s, \tau]} a_{k j}^{[\tau, t]}=\sum_{k=1}^{n}\left(a_{i k}^{[s, \tau]} \sum_{j=1}^{n} a_{k j}^{[\tau, t]}\right)=\sum_{k=1}^{n} a_{i k}^{[s, \tau]} f_{k}(\tau, t), \quad i=1, \ldots, n .
$$

Consequently, using symmetry of the matrix we get

$$
\sum_{i=1}^{n} f_{i}(s, t)=\sum_{k=1}^{n}\left(\sum_{i=1}^{n} a_{i k}^{[s, \tau]}\right) f_{k}(\tau, t)=\sum_{k=1}^{n} f_{k}(s, \tau) f_{k}(\tau, t)
$$

Hence

$$
\sum_{i=1}^{n}\left(f_{i}(s, t)-f_{i}(s, \tau) f_{i}(\tau, t)\right)=0 .
$$

Now we shall derive an equation for functions $g_{i k}$. Using (6.1) from (6.3) we get

$$
g_{i k}(s, t)=\sum_{j=1}^{n} \sum_{p=1}^{n} a_{i p}^{[s, \tau]} a_{p j}^{[\tau, t]}-\sum_{p=1}^{n} a_{i p}^{[s, \tau]} a_{p k}^{[\tau, t]}=\sum_{p=1}^{n}\left(a_{i p}^{[s, \tau]}\left(\sum_{j=1}^{n} a_{p j}^{[\tau, t]}-a_{p k}^{[\tau, t]}\right)\right) .
$$

Hence

$$
g_{i k}(s, t)=\sum_{p=1}^{n} a_{i p}^{[s, \tau]} g_{p k}(\tau, t), \quad i, k=1, \ldots, n .
$$

Using the symmetry of the matrix from the last equality we get

$$
\sum_{i=1}^{n} g_{i k}(s, t)=\sum_{p=1}^{n}\left(\sum_{i=1}^{n} a_{i p}^{[s, \tau]}\right) g_{p k}(\tau, t)=\sum_{p=1}^{n} f_{p}(s, \tau) g_{p k}(\tau, t), \quad k=1, \ldots, n .
$$

Thus we obtain

$$
\sum_{i=1}^{n}\left(g_{i k}(s, t)-f_{i}(s, \tau) g_{i k}(\tau, t)\right)=0, \quad k=1, \ldots, n .
$$

We note that (6.4) and (6.5) are general equations for functions $f_{i}$ and $g_{i k}$. These equations are satisfied, in particular, if

$$
\begin{gathered}
f_{i}(s, t)=f_{i}(s, \tau) f_{i}(\tau, t), \text { for any } i=1, \ldots, n ; \\
g_{i k}(s, t)=f_{i}(s, \tau) g_{i k}(\tau, t), \text { for any } i, k=1, \ldots, n .
\end{gathered}
$$

Equations (6.6) have the following solutions

$$
f_{i}(s, t)=\frac{\Phi_{i}(t)}{\Phi_{i}(s)}, \quad i=1, \ldots, n,
$$


where $\Phi_{i}$ are arbitrary functions with $\Phi_{i}(s) \neq 0$. Substituting this solution in (6.7) we obtain

$$
g_{i k}(s, t)=\frac{\gamma_{i k}(t)}{\Phi_{i}(s)}, \quad i, k=1, \ldots, n,
$$

where $\gamma_{i k}$ are arbitrary functions. By (6.2) and (6.3) we have

$$
a_{i k}^{[s, t]}=a_{k i}^{[s, t]}=f_{i}(s, t)-g_{i k}(s, t)=\frac{\Phi_{i}(t)-\gamma_{i k}(t)}{\Phi_{i}(s)}, \quad i, k=1, \ldots, n .
$$

Thus we have proved the following

Theorem 5. A matrix $\mathcal{M}^{[s, t]}=\left(a_{i k}^{[s, t]}\right)_{i, k=1, \ldots, n}$ given by (6.8) generates an $n$-dimensional $C E A$.

\section{CEAs CORRESPONDING TO BLOCK DIAGONAL MATRICES}

Recall that a block diagonal matrix is a block matrix which is a square matrix, and having main diagonal blocks square matrices, such that the off-diagonal blocks are zero matrices. A block diagonal matrix $M$ has the form

$$
M=\left(\begin{array}{cccc}
M_{1} & 0 & \cdots & 0 \\
0 & M_{2} & \cdots & 0 \\
\vdots & \vdots & \ddots & \vdots \\
0 & 0 & \cdots & M_{m}
\end{array}\right),
$$

where $M_{k}$ is a square matrix. It can also be indicated as $M_{1} \oplus M_{2} \oplus \cdots \oplus M_{m}$.

Lemma 1. Let $\mathcal{M}^{[s, t]}=\mathcal{M}_{1}^{[s, t]} \oplus \mathcal{M}_{2}^{[s, t]} \oplus \cdots \oplus \mathcal{M}_{m}^{[s, t]}$ be a block diagonal matrix. This satisfies (1.2) if and only if each $\mathcal{M}_{i}^{[s, t]}, i=1,2, \ldots, m$ satisfies the equation (1.2).

Proof. It follows from the fact that the product of diagonal matrices amounts to simply multiplying corresponding diagonal elements together.

In [1, [12] it were constructed several CEAs. Using these CEAs and the CEAs constructed in the previous sections, by Lemma 1 one can construct new chains of arbitrary dimensional evolution algebras, i.e. the following is true

Theorem 6. If $\mathcal{M}^{[s, t]}=\mathcal{M}_{1}^{[s, t]} \oplus \mathcal{M}_{2}^{[s, t]} \oplus \cdots \oplus \mathcal{M}_{m}^{[s, t]}$ is a block diagonal matrix, where $\mathcal{M}_{i}^{[s, t]}$ correspond to a known CEA. Then $\mathcal{M}^{[s, t]}$ generates a CEA.

We note that the set of CEAs is very rich: taking matrices $\mathcal{M}^{[s, t]}$ constructed in Theorem 6 as blocks of a new matrix $\tilde{\mathcal{M}}^{[s, t]}$ one can construct new CEAs. Repeating the argument one can construct very rich class of CEAs. 


\section{ACKNOWLEDGEMENTS}

The work is supported by the Grant No.0251/GF3 of Education and Science Ministry of Republic of Kazakhstan.

\section{REFERENCES}

[1] J.M. Casas, M. Ladra, U.A. Rozikov, A chain of evolution algebras, Linear Algebra Appl. 435 (4), 852-870 (2011).

[2] J.M. Casas, M. Ladra, B.A. Omirov, U.A. Rozikov, On evolution algebras, Alg. Colloquim.(to appear)

[3] L.M. Camacho, J.R. Gomez, B.A. Omirov, R.M. Turdibaev, Some properties of evolution algebras, Bulletin of Korean Mathematical Society (to appear).

[4] I.M.H. Etherington, Genetic algebras, Proc. Roy. Soc. Edinburgh. 59, 242-258 (1939).

[5] I.M.H. Etherington, Duplication of linear algebras, Proc. Edinburgh Math. Soc. (2) 6, 222-230 (1941).

[6] I.M.H. Etherington, Non-associative algebra and the simbolism of genetics, Proc. Roy. Soc. Edinburgh. 61, 24-42 (1941).

[7] P. Hänggi, H. Thomas, Time evolution, correlations, and linear response of non-Markov processes, Zeitschrift Phys. B. 26, 85-92 (1977).

[8] S. Karlin, Mathematical models, problems, and controversies of evolutionary theory. Bull. Amer. Math. Soc. (N.S.) 10(2), 221-274 (1984).

[9] Y.I. Lyubich, Mathematical structures in population genetics, Springer-Verlag, Berlin, 1992.

[10] M.L. Reed, Algebraic structure of genetic inheritance, Bull. Amer. Math. Soc. (N.S.) 34(2), 107-130 (1997).

[11] U.A. Rozikov, J. P. Tian, Evolution algebras generated by Gibbs measures, Lobachevskii Jour. Math. 32(4) 270-277 (2011).

[12] U.A. Rozikov, Sh.N. Murodov, Dynamics of two dimensional evolution algebras, arXiv:1202.2690.

[13] J. P. Tian, Evolution algebras and their applications, Lecture Notes in Mathematics, 1921, SpringerVerlag, Berlin, 2008.

[14] A. Wörz-Busekros, Algebras in genetics, Lecture Notes in Biomathematics, 36. Springer-Verlag, Berlin-New York, 1980.

B. A. Omirov and U. A. Rozikov, Institute of Mathematics, National University of UzBekistan, TashKent, UzBekistan.

E-mail address: omirovb@mail.ru rozikovu@yandex.ru

K. M. Tulenbayev, Suleyman Demirel University, Kazakhstan.

E-mail address: tulen75@hotmail.com 